$\mathrm{Z}$ klin Chem. u. klin. Biochem.

8. Jg., S. 595-598, November 1970

\title{
Die Eliminierung von Trübungen bei der Bestimmung von Plasma-Proteinen mit dem Biuret-Reagenz
}

\author{
Von J. P. HofFMANN und R. Richterich \\ Chemisches Zentrallabor des Inselspitals, Bern (Schneiz) und Institut d'Hygiêne et de Santé Publique, Luxembourg
}

(Eingegangen am 17. Juli 1970)

Bei der Biuret-Methode zur Bestimmung der Plasma-Protein-Konzentration war das Problem des Proben-Leerwertes bisher unbefriedigend gelöst. Leerwerte mit Natrium-Chlorid- oder Tartrat-Lösung führen zu eincr systematischen Uber-Korrektur und damit zu zu tiefen Resultaten. Durch Zusatz von Harnstoff in einer End-Konzentration von 6M zum Biuret-Reagenz wird die störende Trübung weitgehend aufgehoben. Das Mitführen eines Proben-Leerwertes erübrigt sich bei der Verwendung eines so modifizierten Reagenzes.

\section{The elimination of turbidity in the determination of plasma proteins with the biuret reagent}

In the determination of plasma protein concentration by the biuret method, the problem of the sample blank value has not hitherto been satisfactorily solved. The use of solutions of sodium chloride or tartrate for the preparation of the blank leads to a systematic over-correction and the results are therefore too low. The addition of urea in a final concentration of $6 \mathrm{M}$ to the biuret reagent largely abolishes the interfering turbidity. With this modified reagent it is unnecessary to run a sample blank.

Die Biuret-Methode $(1,2)$ gilt heute als die zuverlässigste und richtigste Methode zur Bestimmung der Plasma-Proteine (3-6). Als einziger Nachteil sind die Schwierigkeiten mit dem Proben-Leerwert zu erwähnen (4-7), der zur Korrektur von Trübungen und Eigenfarbe des Plasmas mitgeführt werden muß. Es wurde daher versucht, das Biuret-Reagenz so zu modifizieren, daß es zu einer Klärung des Plasmas kommt und der Proben-Leerwert damit überflüssig wird. Dies gelang weitgehend mit einem Biuret-Reagenz, das Harnstoff in einer End-Konzentration von $6 \mathrm{M}$ enthält.

\section{Material und Methoden}

\section{Abkizrzungen}

P (Probe), PL (Proben-Leerwert), RL (Reagenzien-Leerwert), $\mathrm{E}_{\mathrm{abs}}$ (absolute Extinktion), a (prozentualer Extinktions-Koeffizient).

\section{Protein-Bestimmung}

Mit Ausnahme des Harnstoff-Zusatzes lehnt sich die Methode eng an die Modifikation von WeIchselbaum (8) der von KingsLEY (9) beschriebenẹ Technik an (6).

\section{Reagenzien}

Stamm-Lösung (Kalium-Natrium-Tartrat $159 \mathrm{~mm}$, Kupfer-Sulfat $60,1 \mathrm{mM}$, Kalium-Jodid 30,1 mM, Natronlauge $200 \mathrm{~mm})$.

4,5 g Kalium-Natrium-Tartrat $\left(\mathrm{KNaC}_{1} \mathrm{H}_{4} \mathrm{O}_{6}+4 \mathrm{H}_{2} \mathrm{O}\right)$ werden mit $40 \mathrm{ml} 0,2 \mathrm{M}$ Natronlauge gelöst. Unter starkem Rühren $1,5 \mathrm{~g}$ Kupfer-Sulfat $\left(\mathrm{CuSO}_{4}+5 \mathrm{H}_{2} \mathrm{O}\right)$ zusetzen. 0,5 g Kalium-Jodid (KJ) zugeben und mit $0,2 \mathrm{M}$ Natronlauge auf $100 \mathrm{~m} /$ auffüllen. In einer dunklen Flasche bei Raumtemperatur unbeschränkt bältbar.
Verdïnnungs-Lösung
(Kalium-Jodid 30,1 mm, Natronlauge $200 \mathrm{~mm}$ ).

$5 \mathrm{~g}$ Kalium-Jodid $(\mathrm{KJ})$ werden mit $0,2 \mathrm{M}$ Natronlauge auf 1 Liter gelöst. Bei Raumtemperatur unbeschränkt haltbar.

Biuret-Reagenz (Kalium-Natrium-Tartrat $31,8 \mathrm{~mm}$, Kupfer-Sulfat $12,0 \mathrm{~mm}$, Natronlauge $200 \mathrm{~mm}$, Kalium-Jodid $30,1 \mathrm{~mm}$, Harnstoff $6 \mathrm{M})$.

$180 \mathrm{~g}$ Harnstoff werden in einem Gemisch von $100 \mathrm{~m} / \mathrm{Stamm}$ Lösung und $250 \mathrm{~m} l$ Verdünnungs-Lösung aufgelöst. In einem
Meß-Kolben mit Verdünnungs-Lösung auf $500 \mathrm{ml}$ auffüllen. Bei Raumtemperatur einige Wochen haltbar.

Meß-Bedingungen

Kalium-Natrium-Tartrat 31,2 mM, Kupfer-Sulfat 11,8 mM, KaliumJodid 29,5 mM, Harnstoff 5,88 mM, Natronlauge $196 \mathrm{~mm}$; ProbenVolumen (Plasma, Serum) $20 \mu \mathrm{l}$, End-Volumen 1,02 ml; Raumtemperatur, 30 Min.; Messung bei $546 \mathrm{~nm}$ (Albumin : $\mathrm{a}=2,77$ ).

Vorgeben

\begin{tabular}{lcc}
\hline Ansätze & $\mathrm{P}$ & $\mathrm{RL}$ \\
\hline Plasma, Serum, $\mathrm{ml}$ & 0,02 & - \\
demin. Wasser, $\mathrm{m} l$ & $\overline{-}$ & 0,02 \\
Biuret-Reagenz, $\mathrm{ml}$ & 1,00 & 1,00
\end{tabular}

Gut mischen, stehenlassen. Ablesung der Extinktion zwischen 30 und $40 \mathrm{Min}$. bei $546 \mathrm{~nm}$ gegen demineralisiertes Wasser.

Berecbnung $(a=2,77)$

Konzentration $=\frac{E(P)-E(R L)}{a \times d} \times \frac{E V}{A V} g / 100 \mathrm{ml}$

Konzentration $=[\mathrm{E}(\mathrm{P})-\mathrm{E}(\mathrm{RL})] \times 18,4 \mathrm{~g} / 100 \mathrm{~m} l$

Leerwert-Mcssungen

Die Messung des Proben-Leerwertes crfolgte nach 51 facher Verdünnung (20 $\mu \mathrm{l}$ Plasma, $1,0 \mathrm{~m} /$ Leerwert-Lösung) bei $546 \mathrm{~nm}$. Die absolute Extinktion ( $\left.E_{a b s}\right)$ - ergibt sich aus der Formel:

$$
\begin{aligned}
& E_{\mathrm{abs}}=E(P L) \times \text { Verdünnung, } \\
& \mathrm{E}_{\mathrm{abs}}=\mathrm{E}(\mathrm{PL}) \times 51 .
\end{aligned}
$$

Als Proben-Leerwert-Lösungen wurden verwendet: NatriumChlorid-Lösung $154 \mathrm{~mm}$, Tartrat-Natronlauge-Lösung Tartrat $31,2 \mathrm{~mm}$ Natronlauge $196 \mathrm{~ms}$, und Harnstoff-Lösung 6M. Als Untersuchungs-Material diente ausschließlich Plasma, das mit Ammonium-Heparinat antikoaguliert war (6).

\section{Resultate}

Bei allen Versuchen wurde die Extinktion des ProbenLeerwertes nach Verdünnung mit der Natrium-ChloridLösung, der Tartrat-Natronlauge-Lösung und der Harnstoff-Lösung gemessen. Außer den Meß-Extinktionen wurde die absolute Extinktion berechnet sowie 
der Einfluß des Resultates auf eine evtl. Protein-Bestimmung. Diese „Protein-Äquivalente“ wurden durch Multiplikation des gemessenen Proben-Leerwertes mit dem Biuret-Faktor $(18,4)$ erhalten. Die wichtigsten Resultate sind in Tabelle 1 zusammengefaßt.

Tab. 1

Proben-Leerwerte mit Natrium-Chlorid-Lösung, Tartrat- und Harnstoff-Lösung bei der Biuret-Methode zur Protein-Bestimmung. E (PL) Extinktion des Proben-Leerwertes, Eabs absolute Extinktion des Proben-Leerwertes und Protein-Äquivalente in $\mathrm{g} / 100 \mathrm{ml}$. Arithmetisches Mittel und Stancard-Abweichung

\begin{tabular}{|c|c|c|c|}
\hline & $\begin{array}{l}\mathrm{NaCl}- \\
\text { Lösung }\end{array}$ & $\begin{array}{l}\text { Tartrat- } \\
\text { Lösung }\end{array}$ & $\begin{array}{l}\text { Harnstoff- } \\
\text { Lösung }\end{array}$ \\
\hline \multicolumn{4}{|c|}{ I. ",Klare" Plasmen $(\mathrm{N}=42)$} \\
\hline$E(P L)$ & $\begin{array}{c}0,009 \\
(0,002-0,019)\end{array}$ & $\begin{array}{c}0,009 \\
(0,002-0,019)\end{array}$ & $\begin{array}{c}0,006 \\
(0,002-0,015)\end{array}$ \\
\hline $\mathrm{E}_{\mathrm{abs}}$ & $\begin{array}{c}0,459 \\
(0,102-0,060)\end{array}$ & $\begin{array}{c}0,459 \\
(0,102-0,816)\end{array}$ & $\begin{array}{c}0,306 \\
(0,102-0,765)\end{array}$ \\
\hline $\begin{array}{l}\text { Protein-Äq. } \\
\text { g/100 ml }\end{array}$ & $(0,037-0,167$ & $(0,037-0,350)$ & $\begin{array}{c}+0,090 \\
(0,037-0,275)\end{array}$ \\
\hline \multicolumn{4}{|c|}{ II. „Trübe" Plasmen $(N=48)$} \\
\hline$E(P L)$ & $\begin{array}{c}0,028 \\
(0,011-0,047)\end{array}$ & $\begin{array}{c}0,023 \\
(0,009-0,038)\end{array}$ & $\begin{array}{c}0,011 \\
(0,007-0,023)\end{array}$ \\
\hline$E_{a b s}$ & $\begin{array}{c}1,428 \\
(0,561-2,350)\end{array}$ & $\begin{array}{c}1,173 \\
(0,459-1,983)\end{array}$ & $\begin{array}{c}0,561 \\
(0,357-1,173)\end{array}$ \\
\hline $\begin{array}{l}\text { Protein-Äq. } \\
\text { g/100 ml }\end{array}$ & $\begin{array}{c}-0,515 \\
(0,202-0,865)\end{array}$ & $\begin{array}{c}-0,423 \\
(0,167-0,699)\end{array}$ & $\begin{array}{c}+0,202 \\
(0,129-0,423)\end{array}$ \\
\hline
\end{tabular}

In einer 1. Versuchsreihe wurden 42 von Auge ,klarec Plasmen untersucht. Die höchsten Extinktionen des Proben-Leerwertes, nämlich zwischen 0,002 und 0,019 , wurden bei Verdünnung mit der Natrium-ChloridLösung und der Tartrat-Natronlauge-Lösung beobachtet, während bei der Verwendung von HarnstoffLösungen die Extinktionen zwischen 0,002 und 0,015 lagen. Diese Beobachtungen zeigen zunächst, daß Proben mit einer absoluten Extinktion von bis zu 1,0 von Auge nicht als „trüb“ erkannt werden. Sie zeigen weiterhin, daß der Harnstoff-Zusatz die Trübung auf etwa die Hälfte reduziert. Bei der Mitführung eines ProbenLeerwertes und der Verwendung der üblichen Verdünnungs-Lösungen würden die Resultate bis zu $0,35 \mathrm{~g} / 100$ $\mathrm{m} l$ zu tief werden. Bei Verzicht auf einen Proben-Leerwert und Harnstoff-Zusatz ergeben sich Protein-Konzentrationen, die bis zu $0,28 \mathrm{~g} / 100 \mathrm{~m} l \mathrm{zu}$ hoch werden. Noch aufschlußreicher sind die Resultate der 2. Versuchsreihe, bei der 48 von Auge „trübe“ Plasmen analysiert wurden. 24 unausgewählte Resultate der insgesamt 48 Bestimmungen sind in Abbildung 1 dargestellt. Bei der Verdünnung mit Natrium-Chlorid-Lösung lagen die absoluten Extinktionen zwischen 0,56 und 2,35, bei der Verwendung von Tartrat-Natronlauge-Lösung zwischen 0,46 und 1,94 und bei Harnstoff-Zusatz zwischen 0,36 und 1,17. In jedem Fall wurde der ProbenLeerwert nach Harnstoff-Verdünnung um mehr als die Hälfte kleiner als nach der Verdünnung mit den anderen Lösungen. In bezug auf die Protein-Bestimmungen lassen sich folgende Schlüsse ziehen: bei der Durchführung eines Proben-Leerwertes mit Natrium-ChloridLösungen würden die Resultate um bis zu $0,865 \mathrm{~g} / 100$ $\mathrm{m} l$ zu tief, mit Tartrat-Natronlauge-Lösung um bis zu $0,699 \mathrm{~g} / 100 \mathrm{~m} l \mathrm{zu}$ tief, während Harnstoff-Zusatz ohne Mitführen eines Proben-Leerwertes Resultate vortäuscht, die bis zu 0,42 g/100 $\mathrm{m} l$ zu hoch werden. Beim Zusatz

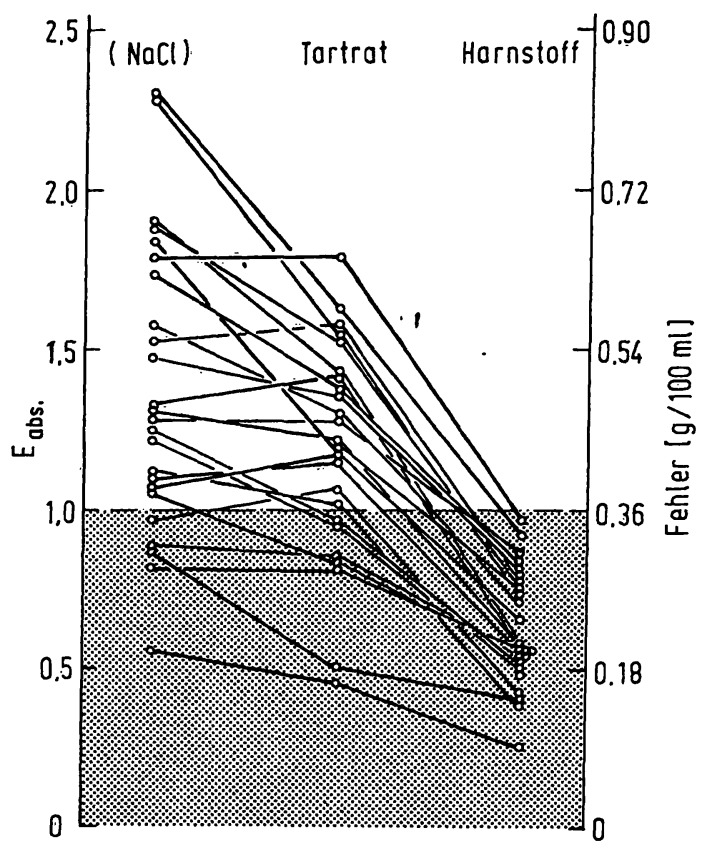

Abb. 1

Extinktionen und entsprechende Protein-Äquivalente in $\mathrm{g} / 100 \mathrm{ml}$ bei der Durchführung von Proben-Leerwerten mit Natrium-Chlorid-, Bestimmungen aus einer Reihe von 48 Analysen von trüben Plasma-
Proben

von Harnstoff fällt auch auf, daß nicht nur eine gewisse Klärung eintritt, sondern daß auch die Streuung der Resultate enger wird. Durch die Verwendung eines etwa 3\% tieferen Biuret-Faktors beim Harnstoff-Zusatz würden die Resultate noch richtiger; in Anbetracht der geringfügigen verbleibenden Trübungen wurde jedoch auf eine Änderung des Biuret-Faktors verzichtet.

Formamid (20 $\mu l$ Plasma $+1,0 \mathrm{ml}$ Formamid) führt ebenfalls zu einer Klärung, die derjenigen des Harnstoffes noch überlegen ist. Hingegen stört Formamid die Biuret-Reaktion (sehr hoher Reagenzien-Leerwert, Verzögerung der Farbentwicklung). Versuche mit Guanidin in Konzentrationen bis zu $6 \mathrm{M}$ ergaben einen geringeren Klär-Effekt als mit Harnstoff.

\section{Diskussion}

Unter Gesamt-Plasma-Proteinen verstehen wir die Summe aller nicht-dialysierbaren (Molekular-Gewicht über 10000) Plasma-Peptide (3,5). Das Charakteristikum dieser Moleküle ist die Peptid-Bindung (10), die auch ihr konstantestes Merkmal in qualitativer und quantitativer Hinsicht darstellt. Im Gegensatz zu älteren Definitionen $(4,11)$ wird der Nicht-Peptid-Anteil, der zwischen $93 \%$ (beim $\alpha_{2}$-Lipoproteid) und $1 \%$ (beim Albumin) liegt, bei der neuen Definition nicht mehr berücksichtigt (3-5). Je nach Definition differieren die Resultate um etwa $10 \%$. Unter Berücksichtigung des Nicht-PeptidAnteiles liegt die Plasma-Protein-Konzentration im Mittel um $7,97 \mathrm{~g} / 100 \mathrm{~m} l$, bei Beschränkung auf den Peptid-Anteil um $6,91 \mathrm{~g} / 100 \mathrm{~m} l$. Die Gründe für das unterschiedliche Verhalten je nach Meß-Methode gehen mit aller Deutlichkeit aus Tabelle 2 hervor.

Höhere Resultate werden mit Methoden erhalten, die das Gesamt-Protein einschließlich des Nicht-Peptid-Anteils 
Der zur Förderung der Forschung gestiftete

\section{HEINRICH - WIELAND - PREIS}

wird hiermit satzungsgemäß für das Jahr 1971 ausgeschrieben.

Der Preis, benannt nach dem 1957 verstorbenen Nobelpreisträger Professor Dr. Heinrich Wieland, ist für Arbeiten aus der Chemie, Biochemie und Physiologie der Fette und Lipoide sowie über deren ernährungsphysiologische und klinische Bedeutung ausgesetzt und wird jährlich verliehen.

Dei HEINRICH-WIELAND-PREIS besteht aus einer "Heinrich-WielandPlakette«, einem Geldbetrag in Höhe von 10000 DM, der Úbernahme aller mit Vervielfältigung und Verteilung der ausgezeichneten Arbeit bis zu einer Auflage von 100 Exemplaren verbundenen Kosten.

Ein Kuratorium, dem zur Zeit die Herren

Prof. Dr. Werner Droese, Dortmund

Prof. Dr. Werner Heimann, Karlsruhe

Prof. Dr. Joachim Kühnau, Hamburg

Prof. Dr. Dr.K. Lang, Bad Krozingen

Prof. Dr. Nepomuk Zöllner, München

angehören, wird den Preisträger auswählen.
Prof. Dr. Rudolf Pannhorst, Berlin

Prof. Dr. Gotthard Schettler, Heidelberg

Prof. Dr. Theodor Wieland, Heidelberg

Prof. Dr. Viktor Wolf, Hamburg

Einsendeberèchtigt für die Verleihung des HEINRICH-WIELAND-PREISES für das Jahr 1971 sind Autoren von unveröffentlichten oder in den Jahren 1969 bis 1971 publizierten wissenschaftlichen Arbeiten. Der eingereichte Beitrag muß in deutscher, englischer oder französischer Sprache abgefaßt sein. Bei fremdsprachlichen Arbeiten ist eine Zusammenfassung (etwa 3 Seiten) in deutscher Sprache erforderlich. Abhandlungen, die bereits mit einem anderen wissenschaftlichen Preis ausgezeichnet sind, können nicht prämiiert werden.

Einsendeșchluß für die Verleihung im Jahre 1971 ist der 1. März 1971.

Die Arbeiten sind in einem Exemplar bis zu diesem Datum an folgende Anschrift einzusenden:

Kuratorium für die Verleihung des HEINRICH-WIELAND-PREISES

Im Auftrag Prof. Dr. Alfons Fricker, 7501 Grötzingen, Ringelberghohl 12 

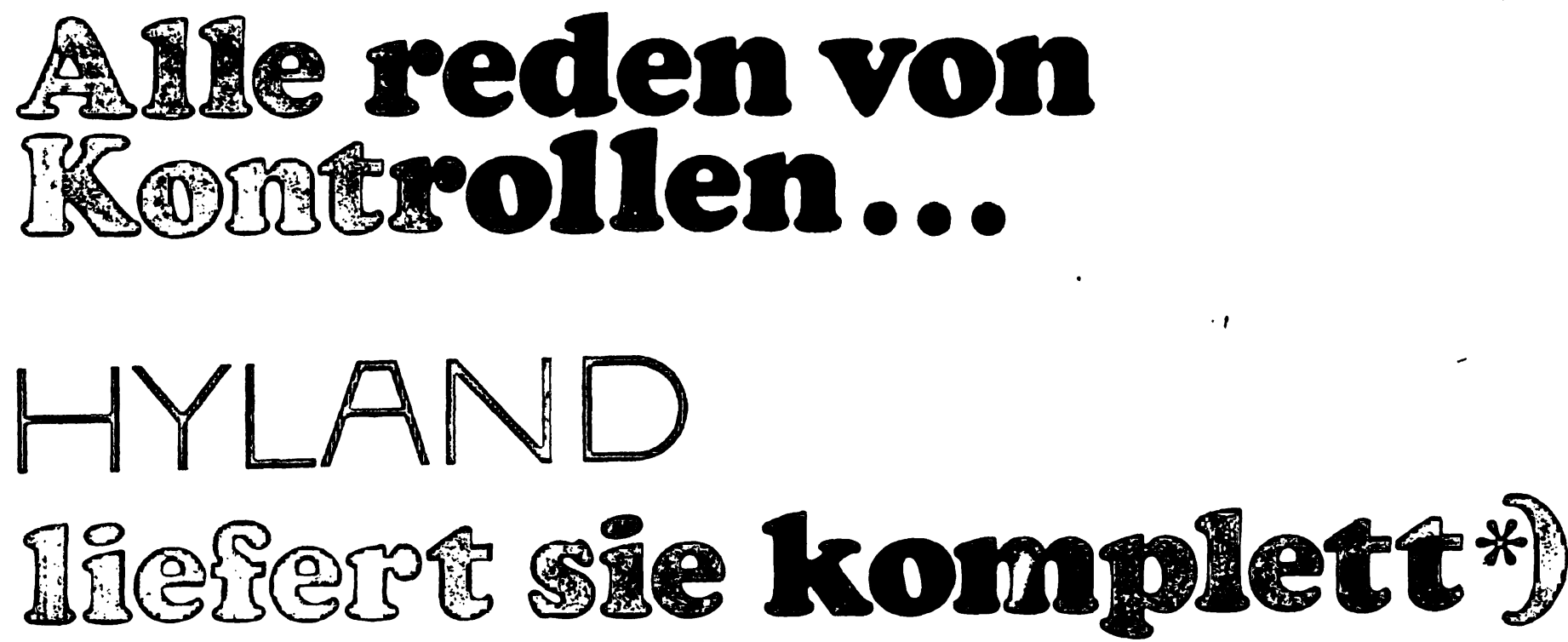

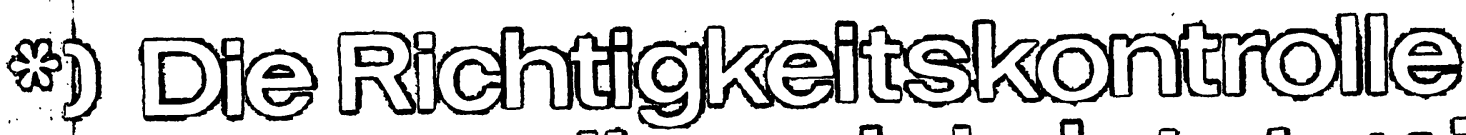

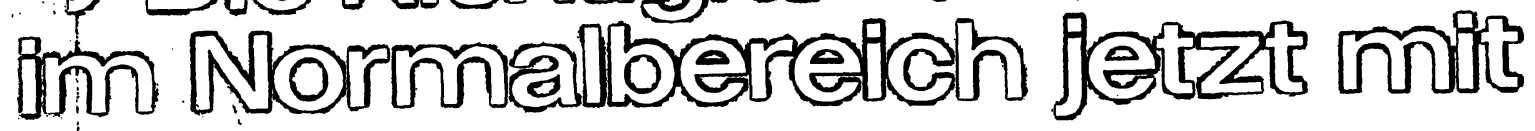

EUROPAWVERTEN

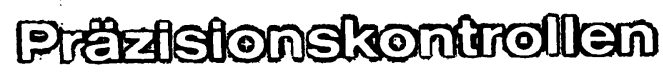

Humanseren, Normal - Pathologisch

Humanserum „Q Pak" für Analyseautomaten

Rilichtigkediskontrollten

Humanseren, Normal - Pathologisch - Spezial

Humanserum "O Pak" für Analyseautomaten

Multi-Enzym-Referenzserum

Bilirubinkontrolle mit erhöhten Werten

Cholesterinkontrolle mit erhöhten Werten

Kontrollurine

Kontroll-Liquor

HB-Referenzstandard und -Kontrolle

Informieren Sie sich bitte. Verlangen Sie unsere Literatur- und Informationsunterlagen.

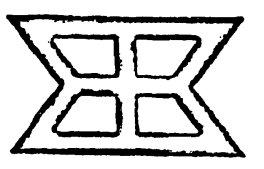

TRAVENOL INTERNATIONAL GMBH

8. München 3, Postfach 113, Tel. (0811) 539376 
Tab. 2

Eigenschaften einiger wichtiger Plasma-Proteide $(5,41)$

\begin{tabular}{|c|c|c|c|c|c|c|c|}
\hline Protein & $\begin{array}{c}\text { Peptid-Anteil } \\
\%\end{array}$ & $\underset{\%}{\text { N-Anteil }}$ & $\begin{array}{l}\text { Kjeldahl- } \\
\text { Faktor }\end{array}$ & $\begin{array}{l}\text { Tyrosin- } \\
\text { Anteil \% }\end{array}$ & $E_{280}^{1 \%}$ & $\underset{\mathbf{a}}{\text { Biuret* }}$ & $\begin{array}{c}\text { Mittlere Plasma- } \\
\text { Konzentration } \\
\text { g/100 ml }\end{array}$ \\
\hline \multirow{2}{*}{$\begin{array}{l}\text { Albumin } \\
\text { Prä-Alburnin } \\
\alpha_{1}-\text { Saures Glykoproteid } \\
\alpha_{1}-\text { Anti-Trypsin } \\
\text { Gc-Globulin } \\
\text { Haptoglobin } \\
\text { Caeruloplasmin } \\
\alpha_{2}-\text { Makroglobulin } \\
\alpha_{2} \text {-Lipoproteid } \\
\text { Transferrin } \\
\text { Hämopexin } \\
\alpha_{2}-\text { Glykoproteid } \\
\text { IgG }\end{array}$} & \multirow{2}{*}{$\begin{array}{l}99,0 \\
97,7 \\
54,9 \\
73,3 \\
90,2 \\
82,2 \\
84,9 \\
84,8 \\
7,3 \\
95,2 \\
70,5 \\
76,3 \\
97,7\end{array}$} & $\begin{array}{l}16,56 \\
16,67 \\
15,24 \\
16,27 \\
16,18 \\
15,40 \\
16,92 \\
16,67\end{array}$ & $\begin{array}{l}6,039 \\
5,999 \\
6,562 \\
6,146 \\
6,180 \\
6,494 \\
5,910 \\
5,999\end{array}$ & $\begin{array}{l}4,07 \\
5,63 \\
7,29 \\
2,55 \\
7,58 \\
6,78 \\
8,90 \\
5,71\end{array}$ & $\begin{array}{r}5,8 \\
13,2 \\
8,9 \\
5,3 \\
12,0 \\
14,9 \\
8,1\end{array}$ & $\begin{array}{r}100,0 \\
97,1 \\
112,0 \\
116,0 \\
105,0 \\
98,0 \\
104,0 \\
107,0\end{array}$ & \multirow{2}{*}{$\begin{array}{l}4,000 \\
0,032 \\
0,087 \\
0,355 \\
0,058 \\
0,110 \\
0,045 \\
0,300 \\
0,190 \\
0,260 \\
0,090 \\
0,023 \\
0,090\end{array}$} \\
\hline & & $\begin{array}{l}17,12 \\
17,56 \\
16,52 \\
16,48\end{array}$ & $\begin{array}{l}5,841 \\
5,695 \\
6,053 \\
6,080\end{array}$ & $\begin{array}{l}4,84 \\
5,30 \\
6,67 \\
6,11\end{array}$ & $\begin{array}{l}11,2 \\
16,9 \\
13,8\end{array}$ & $\begin{array}{r}99,0 \\
112,0 \\
106,0 \\
100,0\end{array}$ & \\
\hline
\end{tabular}

*) in \% von Albumin $(a=2,770)$

messen, so etwa mit der Densitometrie (12) und der Refraktometrie (13, 14). Bei der Bestimmung nach KJELDAHL (15), die den Stickstoff erfaßt, wird meist ein Kompromiß durchgeführt, indem zwar nur der $\mathrm{N}$-Anteil gemessen wird, das Resultat aber mit Hilfe eines Faktors auf die Gesamt-Proteine umgerechnet wird. Dies erklärt z. T. die unterschiedlichen KjeldahlFaktoren: früher 6,25 (16), neuerdings 6,54 (17, 18), bzw. 6,40 (19). Wird reines Plasma-Albumin zur Eichung verwendet, so wird der Faktor 6,04 (5) erhalten. Methoden, die vor allem auf der Messung der aromatischen Ringe (Tyrosin, Tryptophan) beruhen, wie etwa die Technik von Folin-Crocalteu $(20,21)$ und die Messung der Extinktion bei $280 \mathrm{~nm}(22,23)$ sind nicht zu empfehlen, da jedes Protein andere Voraussetzungen aufweist. Hingegen ist die Zahl der Peptid-Bindungen pro Protein-Masse sehr konstant und eignet sich daher vorzüglich als Grundlage für die Messung von ProteinKonzentrationen. Dazu steht einerseits die Messung der Extinktion um $210 \mathrm{~nm}(16,24-26)$ und andererseits die Biuret-Reaktion zur Verfügung. Aus meßtechnischen Gründen wird meist der Biuret-Reaktion der Vorzug gegeben.

Die Biuret-Reaktion, die auf einer Komplex-Bildung eines Kupfer-II-Ions mit 4 Peptid-N-Atomen unter alkalischen Bedingungen (27-29) beruht, ist eine sehr spezifische Reaktion. Außer Peptiden sind wenige Substanzen bekannt, die eine positive Reaktion geben. Praktisch am wichtigsten sind die Amin-Puffer, besonders Tris $(5,30,31)$. Trotz dieser guten theoretischen Fundierung der Spezifität der Biuret-Reaktion darf nicht übersehen werden, daß die Richtigkeit der Methode in der Praxis nicht immer gewährleistet ist. Drei potentielle Fehlerquellen müssen berücksichtigt werden: Der Einfluß von Trübungen, von Plấsma-Farbstoffen und der Hämolyse.

Plasma und Serum sind fast immer trüb, auch wenn von Auge die Trübung kaum erkennbar ist (Tyndall-Effekt). Die oben angeführten Untersuchungen zeigten, daß das menschliche Auge unter den üblichen Labor-Bedingungen Trübungen und Verfärbungen oft erst erkennt, wenn die Extinktion der Probe (bei $546 \mathrm{~nm}$ ) über 1,0 liegt. Eine Eliminierung dieser Trübung durch Verdünnung ist wegen der ungenügenden Empfindlichkeit der Biuret-Methode nicht möglich. Zahlreiche Forscher haben sich denn auch mit dem Problem der Trübung bei der Biuret-Methode beschäftigt und Vorschläge zur Eliminierung dieser Fehlerquelle gemacht:

1. Es wird kein Proben-Leerwert mitgeführt, die Korrektur erfolgt durch einen empirischen, niedrigeren Biuret-Faktor. Ein solches Vorgehen (z. B. Merckotest „Gesamteiweiß") führt zweifcllos in vielen Fällen zu zu hohen Resultaten. Bei extrem lipämischen Plasmen kann das Resultat bis $\mathrm{zu} 8 \mathrm{~g} / 100 \mathrm{ml} \mathrm{zu}$ hoch werden. Auch kann es bei stark lipämischen Proben zu einer Störung der Biuret-Reaktion kommen. Von solchen Verfahren ist daher abzuraten.

2. Es wird ein Proben-Leerwert mit einer Natrium-Chlorid-Lösung durchgeführt (6). Bei einem solchen Vorgehen wird systematisch überkorrigiert. Die Trübung im Proben-Ansatz ist geringer als dicjenige im Leerwert. Dadurch kommt es zu signifikant zu tiefen Resultaten. Wahrscheinlich kommt es während der Biuret-Reaktion zu ciner gewissen Klärung, die in den LeerwertAnsätzen nicht stattfindet. Auch dieses Vorgehen ist daher nicht zu empfehlen.

3. Es wird ein Proben-Leerwert mitgeführt, bei dem als Verdünnungs-Flüssigkeit eine Lösung verwendet wird, die mit dem Biuret-Reagenz identisch ist, jedoch kein Kupfer-Sulfat enthält $(3,32)$. Auch hier sprechen die eigenen Beobachtungen sowie diejenigen von HENRY und Mitarbeitern (7) dafür, daß die Leerwerte systematisch $z u$ hoch werden. Sie sind zwar deutlich niedriger als bei der Verwendung einer Natrium-Chlorid-Lösung, aber noch immer deutlich höher als z. B. bei der Verwendung von Harnstoff zur Klärung. Das Verfahren kann daher nur bedingt empfohlen werden.

4. Die Proteine werden zuerst mit Trichloressigsäure (33) oder Perchlorsäure (6) präzipitiert und anschließend mit Harnstoff oder dem Biuret-Reagenz wieder gelöst. Einem solchen Vorgehen haften zwei Unsicherheiten an: erstens werden Plasma-Farbstoffe zum größten Teil ebenfalls ausgefällt und zweitens werden mit den üblichen Enteiweißungs-Lösungen verschiedene Proteine, z. B. Glycoproteide, nicht oder nicht quantitativ präzipitiert $(38,39)$. Ob dieses Verfahren tatsächlich Vorteile mit sich bringt, ist daher fragwürdig.

5. Von verschiedener Seite wurde ein Ausschütteln mit Äther nach Durchführung der Biuret-Reaktion empfohlen $(4,34)$. Praktisch geht man dabei so vor, daß nach Durchführung der BiuretReaktion $1 \mathrm{~m} /$ Äther zugesetzt, 30 Sek. stark geschüttelt und anschließend scharf zentrifugiert wird. Bei der nachfolgenden Ablesung wird das Resultat etwa $2 \%$ zu hoch $(4,34)$; eine Korrektur für diesen Fehler ist nicht unbedingt notwendig (4). Leider ist es häufig nicht möglich, auf diese Wcise eine Klärung zu erzielen (33). Immerhin dürfte dicses Verfahren bisher noch die besten Resultate gegeben haben.

6. Vereinzelt wurde vorgeschlagen, nach Ablesung der BiuretReaktion den Farbstoff init Kalium-Cyanid $(20 \mathrm{mg} \mathrm{KCN}$ pro $\mathrm{ml}$ 
Biuret-Reagenz) zu entfärben (35) und durch die Messung der Extinktion des farblosen Ansatzes einen Proben-Leerwert zu ermitteln. Wahrscheinlich kommt es aber bei diesem Vorgehen zu einer Uberkorrcktur, d. h. die Resultate werden zu tief (7). Als Notlösung kann dieses Verfahren versucht werden (4).

7. Trübungs-Fehler können mit verschiedenen mathematischen Kunstgriffen eliminiert werden. Ein solches Verfahren wurde auch für die Biuret-Methode angegeben (36). Zuverlässigere Resultate dürfte aber eine Messung bei mehreren Wellenlängen geben (37). In der Praxis sind diese Verfahren zu umständlich.

Wie aus diesen Ausführungen hervorgeht, ist das Problem des Proben-Leerwertes noch nicht gelöst. Die eigenen Untersuchungen haben gezeigt, daß es durch den Zusatz von Harnstoff zu einer weitgehenden Klärung von Plasma-Trübungen kommt. Wird der Harnstoff direkt dem Biuret-Reagenz zugesetzt, so exübrigt sich auch das Mitführen eines Proben-Leerwertes. Bei "klaren“ Plasmen wird der systematische Fehler zu Resultaten führen, die zwischen 0,04 und $0,25 \mathrm{~g} / 100 \mathrm{~m} l$ zu hoch sind. Bei „trüben" Plasmen liegt der Fehler meist zwischen 0,10 und $0,30 \mathrm{~g} / 100 \mathrm{~m} /$. Eine solche $\mathrm{Ab}-$ weichung von der Richtigkeit darf in Kauf genommen werden. Die Abweichung könnte auch durch die Verwendung eines etwa 3\% tieferen Biuret-Faktors fast vollständig eliminiert werden; aus Vergleichsgründen haben wir vorläufig auf die Verwendung eines anderen Faktors verzichtet. Zusammenfassend glauben wir sagen zu dürfen, daß die Verwendung von Harnstoff im Biuret-Reagenz theoretisch und praktisch wahrscheinlich am besten fundiert ist. Als Wirkungs-Mechanismus des Klär-Effektes kann ein Aufbrechen der Sekundär- und Tertiär-Struktur der Proteine postuliert werden. Der verbleibende nicht-klärbare Anteil der Extinktion ist wahrscheinlich nicht durch Trübung, sondern durch die Eigenfarbe der Probe bedingt. Der Vollständigkeit halber sei erwähnt, daß Trübungen, die durch Dextrane (Macrodex, Rheomacrodex) bedingt sind, durch Harnstoff nicht geklärt werden. Bei solchen Plasma-Proben empfiehlt sich eine Zentrifugation vor der Durchführung der Biuret-Reaktion.

Über die Stör-Effekte der Biuret-Reaktion durch die Plasma-Eigenfarbe ist wenig bekannt. Nach Angáben in der Literatur soll Bilirubin bis zu einer Konzentration von $30 \mathrm{mg} / 100 \mathrm{~m} l$ nicht stören $(7,9,40)$.

Beim Vorliegen einer Hämolyse werden die Resultate aus zwei Gründen höher: einerseits gibt Hämoglobin als Protein eine Biuret-Reaktion und andererseits hat Hämoglobin bei $546 \mathrm{~nm}$ eine Eigen-Extinktion. Der Hämoglobin-Fehler läßt sich berechnen. Beim Vorliegen von $100 \mathrm{mg}$ Hämoglobin pro $100 \mathrm{ml}$ Plasma, ergibt das Globin-Molekül eine Protein-Konzentration von $0,1 \mathrm{~g} /$ $100 \mathrm{ml}$. Unter der Annahme, daß überwiegend OxyHämoglobin vorliegt $\left(\varepsilon=14,8 \cdot 10^{3}\right.$, Molekular-Gewicht 16.114) und einer 51fachen Verdünnung, ergibt sich bei $546 \mathrm{~nm}$ eine zusätzliche Extinktion entsprechend $0,33 \mathrm{~g} / 100 \mathrm{ml}$. Hämoglobin in einer Konzentration von $100 \mathrm{mg} / 100 \mathrm{~m} l$ ergibt dadurch theoretisch eine ProteinKonzentration von $0,43 \mathrm{~g} / 100 \mathrm{ml}$. Empirisch wurde gefunden (7), daß $1 \mathrm{mg}$ Hämoglobin $1,9 \mathrm{mg}$ Protein äquivalent sind. In jedem Fall werden die Resultate, wenn von extrem hämolÿtischen Proben (über $1 \mathrm{~g} /$ $100 \mathrm{~m} /$ ) abgesehen wird, nicht wesentlich verfälscht.

Die vorliegende Arbeit wurde $z$. T. durch Unterstützung der Firma Hoffmann-La Roche AG, Basel, Abteilung DIAGNOSTICA, ermöglicht.

\section{Literatur}

1. Rose, F., Poggendorf's Arch. Phys. 28, 132 (1833). - 2. RIEGLER, R., Z. analyt. Chem. 53, 242 (1914). - 3. ReINHold, J. G., Standard Methods in Clinical Chemistry 1, 88 (1953). - 4. HeNRY, R. J., Clinical Chemistry, Principles and Practice, Hoebber and Row Nèw York, N. Y. (1964). - 5. Watson, D., Adv. Clin. Chem. 8, 237 (1965). - 6. RICHTERICH, R., Klinische Chemie, Theorie und Praxis, 2. Aufl. Karger Verlag Basel-New York (1968). - 7. Henrey, R. J., C. Sobex und S. Berkman, Analytic. Chem. 29, 1491 (1957). - 8. Weichselbaum, T. E., Amer. J. Clin. Path. 16, 40 (1946). - 9. KingsLex, G. R., J. Laborat. Clin. Med. S. Louis 27, 840 (1942). - 10. Frscher, E., Sitz.ber. Kgl. Preuß. Akad. Wiss. 1907, p. 35. - 11. Doerr, P., und D. Stamm, diese Z. 6, 304 (1968). 12. Slyke, D. D. van, A. Hiller, R. A. Phillips, P. B. Hamilton, V. P. Dole, R. M. Archibald und H. A. Eder, J. biol. Chemistry 183, 331 (1950). - 13. Remp, D. G., und V. Schelling, Clin. Chem. New York 6, 400 (1960). - 14. Rerss, E., Naunyn-Schmiedebergs Arch. exper. Path. 51, 18 (1904). - 15. KJELDAHL, J., Z. analyt. Chem. 22, 366 (1883). - 16. Goldparb, A. R., L. J. SAIDEL und E. Mosovich, J. biol. Chemistry 193, 397 (1951). - 17. SunderManN, F. W., F. W. Sundermann, E. A. Falvo und C. Kallik, Amer. J. Clin. Path. 30, 112 (1958). - 18. Wolf, A. V., J. B. FulLER, E. J. Goldman und T. D. Mahony, Clin. Chem. New York 8 , 158 (1962). - 19. Watson, D., Clin. chim. Acta Amsterdam 16, 322 (1967). - 20. Folin, O., und V. Crocalteu, J. biol. Chemistry 73, 627 (1927). - 21. Lowry, O. H., N. J. Rosenbrough, A. L. FARR und R. J. Randall, J. biol. Chemistry 193, 265 (1951). 22. Warburg, O., und W. Christian, Biochem. Z. 310,384 (1941).
- 23. Wetlauufer, D. B., Adv. Prot. Chem. 17, 304 (1962). 24. WADDELL, W. J., J. Laborat. Clin. Med. S. Louis 48, 311 (1956). -25. Tombs, M. P., F. Souter und N. F. Maclagan, Biochem. J. 73, 167 (1959). - 26. Watson, D., Clin. Chem. New York 10, 412 (1964). - 27. FreEMAN, H. C., J. E. W.L. SMITH und J. C. TAYLor, Nature London 184, 707.(1959). - 28. KLOTZ, I. M., I. L. FALLER und J. M. Urquhart, J. Phys. Coll. Chem. 54, 18 (1950). - 29. MehL, J. W., E. Pacovska und R. J. WInzzLeR, J. biol. Chemistry 177, 13 (1949). - 30. Stewart, L. E., J. W. Thomas und G. E. Hurc, Clin. chim. Acta Amsterdam 44, 453 (1949). - 31. Robson, R. M., D. E. Goll und M. J. Temple, Analytic. Biochem. 24, 339 (1968) - 32. Watson, D., und I. Farrance, Proc. Australian Ass. Clin. Biochem. 1, 87 (1946). - 33. Robinson, H. W., und C. G. Hodgen, J. biol. Chemistry 135, 707 (1940). - 34. KINGsLEY, G. R., J. biol. Chemistry 131, 197 (1939). - 35. KEYSER, J. W., und J. VAUGH, Biochem. J. 44, xxii (1949), Bode, Ch., H. Goebell und E. StÄHLER, diese Z. 6, 481 (1968). - 36. INCHrosA, M. A., J. Laborat. Clin. Med. S. Louis 63, 319 (1964). - 37. FoG, J., Scand. J. Clin. Laborat. Invest. 1, 255 (1949). - 38. ROBERT, B., C. DE VAUXSt. Cyr, L. Robert und P. Grabar, Clin. chim. Acta Amsterdam 4, 828 (1959). - 39. Winzler, R. J., A. W. Devor, J. W. Mehl und I. M. SMYThe, Analytic. Chem. 18, 229 (1946). - 40. Gornald, A. G., C. J. Bardawirl und M. M. David, J. biol. Chemistry 177, 751 (1949). - 41. Schultze, H. E. und J. F. Heremans, Molecular Biology of Human Proteins, Vol. I Elsevier, New York (1966).
Prof. Dr. R. Richterich Chemisches Zentrallabor Inselspital CH 3008 Bern 